\title{
Normalização da força e torque muscular em crianças e adolescentes
}

\section{Normalization of muscle strength and torque in children and adolescents}

1 Universidade Federal do Rio Grande do Sul. Escola de Educação Física. Laboratório de Pesquisa do Exercício. Porto Alegre, RS. Brasil

2 Faculdade Sogipa de Educação Física. Porto Alegre, RS. Brasil

Recebido em 15/02/11 Revisado em 13/05/11 Aprovado em 15/06/11
Resumo - Os métodos tradicionais de normalização dos dados de força muscular (FM) e torque muscular (TM) têm sido considerados inadequados para ajustar apropriadamente os efeitos da massa corporal em crianças e adultos. A alometria tem demonstrado ser o método mais eficiente para comparar indivíduos de diferentes dimensões corporais. Entretanto, os valores que o expoente alométrico pode assumir para FM e TM ainda são controversos e dependem de diversos fatores relacionados à composição corporal, sexo, idade, tipo de contração e modalidade esportiva. $\mathrm{O}$ volume muscular (VM), atualmente, tem demonstrado ser a variável emergente para a normalização dos dados de FM e TM.

Palavras-chave: Crianças; Força muscular; Torque.

Abstract - Traditional methods of normalization of muscle strength (MS) and muscle torque (MT) have been considered inadequate to properly adjust the effects of body mass in children and adults. Allometry has proved to be the most efficient method to compare individuals of different body dimensions. However, the values that the allometric exponent may show for MS and MT are still controversial and depend on many factors related to body composition, sex, age, type of contraction, and type of sport. Muscle volume (MV) has been suggested as the emerging variable for normalization of MS and MT.

Key words: Muscle strength; School children; Torque. 


\section{INTRODUÇÃO}

O treinamento de força tem sido considerado efetivo e seguro para o desenvolvimento da força muscular (FM) em crianças e adolescentes ${ }^{1}$. Testes de FM têm sido extensivamente utilizados no esporte e na educação física com o objetivo de fornecer valores normativos (perfil atlético), detecção de talentos, distinção entre diferentes níveis de desempenho ou avaliar adaptações ao treinamento ${ }^{2}$. A FM tem um papel importante para o desempenho esportivo, pois melhora a velocidade de execução de habilidades técnicas específicas e também é importante para estabilizar as articulações em diversas situações esportivas, auxiliando na prevenção de lesões².

Durante a infância e a adolescência, a FM absoluta apresenta um aumento progressivo ${ }^{3,4}$. Esse aumento é atribuído, principalmente, a mecanismos neurais, como o aumento no recrutamento e na frequência de ativação de unidades motoras ${ }^{1}$. Contudo, durante o processo de maturação biológica, modificações no tamanho, massa e composição corporal influenciam esse comportamento da $\mathrm{FM}^{4-6}$. Por esse motivo, tem sido postulado que o melhor período para o treinamento e desenvolvimento da FM seria durante o processo maturacional. Especificamente, o melhor período para o desenvolvimento da FM seria de 12-18 meses após o pico de velocidade da estatura para meninos e, imediatamente após a menarca, para meninas?

Entretanto, essa teoria deve ser interpretada com cautela, pois quando mensuramos a FM na população pediátrica, a idade, o sexo, a maturação biológica e o tamanho corporal são variáveis intervenientes. Assim, esse aumento da FM observado durante o processo maturacional poderia ser um efeito de escala, resultado de uma incorreta normalização dos dados. Desta forma, é extremamente importante que o efeito do tamanho corporal sobre os dados de FM e torque muscular (TM) seja devidamente normalizado. Caso contrário, não será possível definir se as alterações na FM e TM são devidas ao crescimento, à maturação biológica, ao treinamento, à composição corporal ou a uma interação entre esses fatores.

Historicamente, esse efeito do tamanho corporal sobre a FM e TM tem sido controlado por uma simples divisão do valor da FM absoluta pela massa corporal (MC) em quilogramas (kg), conhecida como expressão padrão (FM/MC). A MC foi escolhida como variável de normalização porque geralmente apresenta altos valores de correlação com a FM e TM. Entretanto, nos últimos anos, esse método de normalização tem sido extensamente criticado, pois a relação entre MC e FM não tem demonstrado ser linear, com os valores de FM aumentando em uma menor proporção do que a $\mathrm{MC}^{8-11}$. Caso esse comportamento seja observado, a expressão padrão torna-se inapropriada para ajustar adequadamente os efeitos da MC sobre a FM e TM.

$\mathrm{Na}$ tentativa de realizar comparações adequadas entre indivíduos heterogêneos em tamanho corporal, outras variáveis de normalização da FM e TM têm sido propostas, como a utilização do índice de massa corporal (IMC), massa livre de gordura (MLG), área de secção transversa (AST) e volume muscular (VM) ${ }^{3,12-14}$. Adicionalmente, muitos estudos têm indicado a alometria como método mais apropriado para ajustar os efeitos da MC sobre a $\mathrm{FM}^{15-18}$ ou $\mathrm{TM}^{19-24}$. Essa metodologia é baseada na aplicação da equação alométrica $\left(\mathrm{Y}=\mathrm{aX}{ }^{\mathrm{b}}\right)$, que estabelece um índice que permite comparar indivíduos heterogêneos em tamanho e composição corporal.

Por todo o exposto, a presente revisão tem como objetivo apresentar uma revisão crítica em relação aos métodos de normalização dos dados de FM e TM em crianças e adolescentes, bem como sugerir recomendações para a normalização dessas variáveis.

\section{PROCEDIMENTOS METODOLÓGICOS}

Os artigos dessa revisão foram selecionados a partir das bases de dados PubMed e Scopus. Foram incluídos artigos publicados na íntegra, no período entre 1980 e 2011, que apresentavam as palavras chave allometric, strength, torque, children, normalizing, biological maturation e suas possíveis combinações. Fontes adicionais foram obtidas através das referências encontradas nos artigos primários.

\section{VARIÁVEIS TRADICIONAIS DE NORMALIZAÇÃO DA FM E TM}

A FM tem sido definida como a força máxima $(\mathrm{N})$ ou o torque máximo $(\mathrm{Nm})$ desenvolvido durante uma contração voluntária máxima $(\mathrm{CVM}) \mathrm{em}$ dada condição ${ }^{18}$.

Diversos fatores afetam os resultados de FM e TM. Entre eles, podemos citar fatores relacionados aos participantes (sexo, idade, estágio maturacional, composição corporal e nível de condicionamento físico) $^{5,6}$, à arquitetura muscular (ângulo de penação, comprimento do fascículo, espessura muscular e $\mathrm{VM})^{13,14}$, a aspectos metodológicos (tipo de contra- 
ção muscular, estabilidade, correção da gravidade e sequência do protocolo de teste) $)^{5,18}$, a fatores neuromusculares (ativação e coativação de agonistas e antagonistas) $)^{5,12}$, e outros relacionados à inconsistência das variáveis utilizadas para a normalização dos dados (MC, IMC, MLG, AST e VM) 3,12-14.

Embora os efeitos do tamanho e da composição corporal sobre os valores de FM e TM sejam conhecidos, a normalização dos dados tem sido inconsistente. Por exemplo, alguns estudos têm apresentado dados de FM não normalizados pelo tamanho corporal ${ }^{25,26}$, normalizados por expressões padrões (FM/estatura, FM/IMC, FM/MLG) ou normalizados por vários métodos diferentes para a mesma série de dados (MC, MLG, AST e VM) $)^{12,27}$.

Outras maneiras de normalizar os dados de FM e TM têm sido baseadas na teoria da similaridade geométrica, em que se afirma que o corpo humano tem a mesma forma, variando somente em tamanho corporal. Dessa forma, a FM deveria ser proporcional à estatura ao quadrado $\left(E^{2}\right)$. Assim, qualquer área de um objeto geometricamente similar é, em princípio, proporcional ao volume corporal ou a MC elevados na potência de dois ter$\operatorname{ços}\left(\mathrm{V}^{2 / 3}, \mathrm{MC}^{2 / 3}\right)^{19}$. Entretanto, as descobertas dessa teoria, que foram baseadas a partir de um músculo hipotético, têm sido estendidas diretamente para a normalização dos dados de FM e TM, o que dificulta ainda mais a comparação dos resultados de diferentes estudos.

Por muitos anos tem sido postulado que a produção de FM é diretamente dependente da AST e, por esse motivo, a normalização da FM pela AST (FM/AST) tem sido considerada o método padrão ouro $^{12,28,29}$. Entretanto, a normalização FM/AST também tem demonstrado ser inconsistente. Algumas justificativas poderiam ser atribuídas aos diferentes métodos de determinação da AST, ao sexo, à idade e ao nível de condicionamento físico, mas, principalmente, porque essa relação direta entre FM e AST muitas vezes não tem sido observada ${ }^{16,29}$. Para exemplificar essa inconsistência, Maughan et al. ${ }^{30}$ compararam a FM/AST nos extensores do joelho em dois grupos de atletas de elite (velocistas e maratonistas) e um grupo controle. Os resultados revelaram que os velocistas $\left(9,9 \pm 0,9 \mathrm{~N} / \mathrm{cm}^{2}\right)$ eram significativamente $(\mathrm{p}<0,01)$ mais fortes do que os maratonistas $\left(8,8 \pm 0,5 \mathrm{~N} / \mathrm{cm}^{2}\right)$ e semelhantes ao grupo controle $\left(9,5 \pm 1,4 \mathrm{~N} / \mathrm{cm}^{2}\right)$, não existindo diferenças na AST entre os grupos. $\mathrm{O}$ resultado mais curioso foi que os sujeitos do grupo controle apresentaram maiores valores individuais de FM/AST do que o grupo de atletas $\left(13,6 \mathrm{~N} / \mathrm{cm}^{2}\right.$ vs $\left.11,4 \mathrm{~N} / \mathrm{cm}^{2}\right)$. Especificamente em crianças, a AST não tem demonstrado ser uma variável explicativa da $\mathrm{FM}^{3}$, além de não aumentar na mesma proporção que a $\mathrm{FM}^{5}$.

$\mathrm{Na}$ tentativa de encontrar um método que permita comparar adequadamente indivíduos de diferentes idades, sexo e composição corporal, diversos estudos têm indicado a alometria como método de normalização mais adequado para os dados de FM e TM ${ }^{10,18,21,23}$. O quadro 1 apresenta, em resumo, os principais estudos que utilizaram a alometria para normalização os dados de FM e TM.

\section{UTILIZAÇÃO DA ALOMETRIA PARA NORMALIZAÇÃO DE DADOS BIOLÓGICOS}

A alometria é o método matemático que expressa a medida à qual uma variável (seja anatômica, fisiológica, física ou temporal) é relacionada com uma unidade de tamanho corporal ${ }^{4}$.

A análise alométrica pode ser descrita pela equação $Y=a X^{b}$, em que $Y$ é a variável dependente e X a variável independente (geralmente $\mathrm{MC}$ em $\mathrm{kg}$ ). Essa equação possui dois termos importantes, o coeficiente de proporcionalidade "a" e o expoente alométrico "b". Esses dois termos podem ser identificados por meio de uma análise de regressão linear após convertermos os valores de $\mathrm{X}$ e $\mathrm{Y}$ para logaritmo. Assim, temos a equação $\log \mathrm{Y}=\log \mathrm{a}+\mathrm{b}$ $\log \mathrm{X}$. O coeficiente de proporcionalidade "a" pode ser utilizado para comparar a magnitude das forças. O expoente alométrico "b" indica a extensão e a direção da relação entre a variável dependente $Y$ e a variável independente $\mathrm{X}$. $\mathrm{O}$ expoente alométrico "b" pode apresentar diferentes valores, tanto positivos como negativos, dependendo da relação a ser observada. Além disso, caracteriza de forma descritiva a relação entre as alterações da variável dependente $\mathrm{Y}$ e a variável independente $\mathrm{X}^{31}$.

Do ponto de vista esportivo, muitos estudos têm utilizado a alometria para normalizar diversas variáveis, como o $\mathrm{VO}_{2}$ max e limiar anaeróbio ${ }^{32,33}$, FM e TM ${ }^{8,16,18,27}$ e efeitos da maturação biológi$\mathrm{ca}^{13,14,32,33}$. Entretanto, poucos estudos têm examinado diretamente a aplicação dos expoentes alométricos durante o crescimento e maturação biológica de crianças, adolescentes e jovens atletas.

\section{UTILIZAÇÃO DA ALOMETRIA PARA A NORMALIZAÇÃO DOS DADOS DE FM E TM}

Algumas investigações têm indicado que a FM aumenta em uma menor proporção do que o ta- 
Quadro 1. Resumo dos estudos que utilizaram a alometria para normalização dos dados de força e torque muscular.

\begin{tabular}{|c|c|c|c|}
\hline Estudo & Participantes & Teste & Comentários \\
\hline Davies et al..$^{34}$ & Idosos $(n=148)$ & TM de EJ & Recomenda MLG para a normalização. \\
\hline De Ste Croix et al. ${ }^{3}$ & $\begin{array}{l}\text { o e }+10-14 \\
\text { anos }(n=41)\end{array}$ & TM de EJ e FJ & $\begin{array}{l}\text { Sem diferença }(\mathrm{p}<0,05) \text { entre } \hat{o} \mathrm{e} \text { } \\
\text { quando a } M C \text { é ajustada por alometria. }\end{array}$ \\
\hline De Ste Croix et al. ${ }^{35}$ & $\begin{array}{l}\delta \text { e } q 8-9 \\
13-14 \text { e } 18-27 \\
\text { anos }\end{array}$ & TM de EJ e FJ & $\begin{array}{l}\text { "b" varia com a idade, e, após a normalização, } \\
\text { verificou-se diferenças entre os grupos. }\end{array}$ \\
\hline Folland et al..$^{23}$ & $\begin{array}{c}18-30 \text { anos } \\
(n=86)\end{array}$ & TM e FM de EJ & $\begin{array}{c}\text { Valores de "b" são menores para a FM e para } \\
\text { indivíduos não-atletas. }\end{array}$ \\
\hline Hullens et al..$^{27}$ & $\begin{array}{c}\text { + obesas e } \\
\text { não-obesas } \\
(\mathrm{n}=253)\end{array}$ & $\begin{array}{c}\text { TM de EJ, FJ, tronco e } \\
\text { FPM }\end{array}$ & $\begin{array}{l}\text { A alometria verificou que } \text { o não-obesas } \\
\text { têm maiores valores de FM e TM. }\end{array}$ \\
\hline Jaric et al. ${ }^{9}$ & $\begin{array}{c}22-47 \text { anos } \\
(\mathrm{n}=16)\end{array}$ & $\begin{array}{l}\text { FM, TM de EJ, FJ, EC, } \\
\text { FC, EQ, FQ }\end{array}$ & $\begin{array}{l}\text { Recomenda a alometria para normalizar FM } \\
\qquad(b=0,67) \text { e TM }(b=1) .\end{array}$ \\
\hline Markovic et al. ${ }^{21}$ & $\begin{array}{c}18-26 \text { anos } \\
(\mathrm{n}=77)\end{array}$ & FM e potência & $\begin{array}{c}\text { Aplicação de diferentes "b" para normalizar FM } \\
\text { (carga externa e interna); a potência não necessi- } \\
\text { ta de normalização pela MC. }\end{array}$ \\
\hline Nevill et al..$^{10}$ & $\begin{array}{c}\text { o e } 11-16 \\
\text { anos } \\
(n=453)\end{array}$ & TM de EJ e FC & $\begin{array}{c}\text { A alometria identificou efeito positivo } \\
\text { adicional da idade cronológica sobre o } \\
\text { TM em } \delta \text {. }\end{array}$ \\
\hline Vanderburg et al. ${ }^{24}$ & o e $q(n=205)$ & FPM & $\begin{array}{l}\text { Recomenda normalizar FPM por } \\
\text { "b" }=0,54 \text { para }{ }^{\lambda} \mathrm{e} " \mathrm{~b} "=0,48 \text { para } q .\end{array}$ \\
\hline Weir et al. ${ }^{11}$ & $\begin{array}{l}\text { Lutadores de } 8-13 \text { e } 14- \\
\quad 18 \text { anos }(\mathrm{n}=258)\end{array}$ & TM EJ e FJ & $\begin{array}{l}\text { TM aumenta com a idade e não pode ser expli- } \\
\text { cado somente pelo aumento da MLG. }\end{array}$ \\
\hline Wisloff et al. ${ }^{8}$ & Futebolistas $(\mathrm{n}=29)$ & Levantamento de peso & Recomenda normalizar a FM por "b" $=0,67$. \\
\hline Wren et al..$^{17}$ & $\begin{array}{c}\text { e }+4-16 \\
\text { anos } \\
(n=39)\end{array}$ & $\begin{array}{l}\text { TM de EJ, FJ, ADC, } \\
\text { ABC, FPT e FDT }\end{array}$ & $\begin{array}{l}\text { Normalização padrão falhou em ajustar o TM; } \\
\text { recomenda o uso da alometria. }\end{array}$ \\
\hline Zoeller et al..$^{16}$ & $\begin{array}{l}\text { 23-26 anos } \\
(\mathrm{n}=136)\end{array}$ & FM isométrica de FC & $\begin{array}{l}\text { Normalização alométrica removeu os efeitos da } \\
\text { MC sobre os dados de FM isométrica (" } b \text { " }=0,64 \text { ). }\end{array}$ \\
\hline
\end{tabular}

$\mathrm{FM}=$ força muscular; $\mathrm{TM}=$ torque muscular; $\mathrm{EJ}=$ extensores do joelho; $\mathrm{FJ}=$ flexores do joelho; $\mathrm{FC}=$ flexores do cotovelo; $\mathrm{EC}=$ extensores do cotovelo; $\mathrm{ADC}=$ adutores da coxa; $\mathrm{ABC}=$ abdutores da coxa; $\mathrm{FPT}=$ flexores plantares do tornozelo; $\mathrm{FDT}=\mathrm{Flexores}$ dorsais do tornozelo; " $\mathrm{b}$ "= expoente alométrico; $\mathrm{MC}=$ massa corporal; $\mathrm{FPM}=$ força de preensão manual; $\mathrm{AST}=$ área de secção

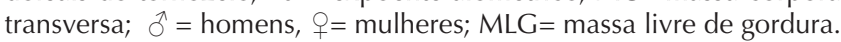

manho corporal ${ }^{8-11}$. Por esse motivo, os modelos alométricos têm sido utilizados para ajustar adequadamente os efeitos do tamanho corporal sobre os dados de FM e TM ${ }^{10,11,18,23}$. A identificação do expoente alométrico nos permite identificar a direta relação entre a FM ou TM com a sua variável de normalização e estabelece um índice independente do tamanho corporal.

No entanto, o valor que esse expoente alométrico (b) pode assumir tem apresentado uma grande variação, pois é dependente do tamanho amostral, da variável de normalização (MC, MLG, AST, ASTF e VM) e da forma de manifestação da força (isométrica, concêntrica e excêntrica). Nesse sentido, exercícios de FM que necessitam vencer uma resistência externa (ex.: preensão manual, agachamento, supino, rosca bíceps e tríceps) geralmente apresentam expoentes alométricos de aproximadamente $b=0,67$. Exercícios que suportem o peso corporal (ex.: abdominal, barra, apoio e elevação de pernas) apresentam valores negativos para b=-0,33; e exercícios de FM com componente de potência (ex.: salto vertical, salto horizontal, chute e arremessos) têm valores de $b=0$, ou seja, sem necessidade de normalização pela $\mathrm{MC}^{21}$.

Outro problema reportado é a distinção entre as formas de normalização para a FM e o TM9 . Essa distinção é necessária, pois o torque é uma função da FM, com a vantagem biomecânica do sistema de alavanca ${ }^{5}$. As evidências demonstram que os métodos de normalização permanecem semelhantes para FM e $\mathrm{TM}^{27,36}$, causando problemas para a normalização dos efeitos do tamanho corporal. Por outro lado, se os indivíduos testados forem geometricamente similares, o efeito da alavanca não dependerá do tamanho corporal, desde que o braço de alavanca varie proporcionalmente.

Jaric et al. ${ }^{9}$ propuseram a utilização de diferentes expoentes alométricos para normalizar a FM $(b=0,67)$ e o TM $(b=1)$. Esses autores teorizaram que o tamanho corporal pode alterar o braço de alavanca muscular e a resistência externa poderia 
variar na mesma proporção. Dessa forma, a força gerada poderia ser proporcional a FM, visto que esta é dependente da AST do músculo, que, por sua vez, é proporcional à $\mathrm{MC}^{0,67}$. Ao contrário, o TM depende da FM multiplicada pelo braço de alavanca do músculo (BAM), que é proporcional à $\mathrm{MC}^{0,33}$. Em vista disso, podemos expressar essas relações como TM= FM x BAM, ou seja, $\mathrm{MC}^{0,67} \times \mathrm{MC}^{0,33}=$ MC1. Assim, o expoente alométrico $b=1$ deveria ser utilizado para normalizar os dados de TM, o que teoricamente torna a normalização padrão (TM/ MC) adequada. Entretanto, alguns estudos têm demonstrado que esse expoente alométrico pode variar de $b=0,77$ até $b=1,87$ em resposta a diferentes protocolos isocinéticos ${ }^{10,17,35,36}$.

$\mathrm{O}$ valor que o expoente alométrico pode assumir para a FM e TM é outro fator que reforça a necessidade de distinção na normalização dessas variáveis. Geralmente, a FM tem apresentado menores valores de expoente alométrico do que o $\mathrm{TM}^{27}$. Vanderburgh et al. ${ }^{24}$ sugerem valores de expoente alométrico para a FM que variam de $0,48<b<0,58$, enquanto que Jaric et al. ${ }^{9}$ recomendam valores de $b=0,67$. Estudos com levantamento de pesos têm apresentado uma maior variação dos expoentes alométricos para FM $(0,45<b<0,87)$. Quando analisamos o TM, esse tem apresentado consistentemente maiores valores de expoente alométrico $(0,74<\mathrm{b}<1,31)^{9,11,27,34}$.

Na tentativa de elucidar esses questionamentos, Folland et al. ${ }^{23}$ verificaram as diferenças entre FM isométrica e o TM dos extensores do joelho em 86 homens. Os resultados demonstraram que o expoente alométrico para o TM $(b=0,69)$ foi consistentemente maior do que para a FM $(b=0,33)$. Quando a amostra foi separada em dois grupos de acordo com o percentual de gordura (menor e maior do que 20\%), os expoentes alométricos correspondiam a b=0,45 e $b=0,68$ para $F M$, e $b=0,68$ e $b=0,98$ para $T M$, respectivamente. Quando a MLG foi utilizada como uma variável de normalização dos dados, essa igualmente evidenciou maiores valores de expoente alométrico para o TM $(b=1,12)$ do que para a FM $(b=0,76)$.

Excepcionalmente, Nevill et al. ${ }^{10}$ apresentaram valores de expoente alométrico bem abaixo aos reportados pela literatura para o TM de bíceps e quadríceps ( $b=0,38$ e $b=0,36$, respectivamente). Entretanto, a amostra desse estudo foi composta de crianças, e os resultados podem ter sido afetados pelo efeito da maturação biológica.

\section{UTILIZAÇÃO DA ALOMETRIA PARA NORMALIZAÇÃO DA FM E TM EM}

\section{CRIANÇAS E ADOLESCENTES}

A alometria tem sido utilizada para normalizar os dados de FM e TM em crianças e adolescentes, principalmente, porque permite identificar um índice estatisticamente independente do tamanho corporal. A identificação desse índice é extremamente importante, pois os dados de FM e TM também são influenciados pela idade cronológica, sexo, treinamento e composição corporal.

Do ponto de vista do treinamento desportivo, tem sido postulado que o melhor período para desenvolver a FM seria durante o processo maturacional? . Essa indicação é baseada principalmente em estudos que identificaram um aumento progressivo da FM absoluta (sem normalização) de acordo com a idade cronológica e maturação biológica. A partir disso, diversos estudos utilizaram a alometria para normalizar adequadamente os dados de FM e TM, na tentativa de elucidar o real comportamento da FM em crianças e adolescentes $3,10,24,35$.

Um dos primeiros estudos a utilizar a alometria para normalizar os dados de FM em crianças foi o de Vanderburgh et al. ${ }^{24}$. Esse estudo tinha como objetivo identificar um índice que permitisse comparar a força de preensão manual entre meninos $(n=100)$ e meninas $(n=105)$ independentemente dos efeitos de confusão da MC. Os autores concluíram que o expoente alométrico $b=0,51$ normalizava adequadamente os dados e permitia uma comparação entre meninos e meninas independente da $\mathrm{MC}\left(\mathrm{FM} / \mathrm{MC}^{0,51}\right)$.

De Ste Croix et al. ${ }^{35}$ examinaram os efeitos da idade e do sexo sobre a FM isocinética de membros inferiores em meninos, meninas e adultos. Foram identificados os expoentes alométricos para o TM de extensores do joelho $(b=0,82$ e $b=0,85$ para homens e mulheres, respectivamente) e para o TM de flexores do joelho $(b=1$ e $b=0,96$ para homens e mulheres, respectivamente). Os resultados demonstraram que não existiam diferenças no TM absoluto (TM), relativo (TM/MC) e alométrico (TM/MC $\left.{ }^{b}\right)$ entre crianças e adolescentes, mas existiam diferenças entre os sexos no grupo dos adultos. Os dados sugerem que quando a MC é devidamente ajustada por alometria, a FM isocinética aumenta de acordo com a idade, e as diferenças entre os sexos não são evidentes até os 14 anos.

Wren et al. ${ }^{17}$ verificaram a aplicação de diversos métodos de normalização do TM de membro inferior em crianças. Foram utilizadas as normalizações TM/MC, TM/MCxE, TM/IMC e o modelo alométrico TM/MCb. Os resultados demonstraram 
que a simples normalização (TM/MC) não ajusta adequadamente os valores de TM em crianças. $\mathrm{O}$ modelo alométrico demonstrou ser o método mais apropriado para a normalização do TM, sendo que os valores dos expoentes alométricos variaram de $\mathrm{b}=1,32$ até $\mathrm{b}=1,87$, com um valor médio de $\mathrm{b}=1,60$ para os seis movimentos testados. Outro estudo utilizou a alometria para examinar a influência da MLG sobre o TM em dois grupos de levantadores de peso, classificados por idade cronológica (8-13 anos vs 14-18 anos). Os resultados indicaram que a TM aumentava de acordo com a idade cronológica e não poderia ser explicado pelo aumento da MLG. $\mathrm{O}$ efeito da idade cronológica foi maior no grupo 8-13 anos e poderia ser devido ao desenvolvimento do sistema neuromuscular, alterações na massa muscular ou a combinação desses fatores ${ }^{11}$.

Assim como a idade cronológica, a maturação biológica é considerada uma variável crítica quando analisamos a FM e o TM em crianças e adolescentes, pois o desenvolvimento do sistema nervoso e a mielinização neuromuscular só estarão totalmente completos ao final desse processo. Estudos examinando os efeitos da maturação biológica sobre a FM devem ser interpretados com muita cautela, pois esses estimam o estágio maturacional a partir da idade cronológica ou simplesmente classificam os indivíduos como sendo pré-púberes ou pós-púberes 5 . Adicionalmente, existe uma dificuldade de dissociar os efeitos da maturação biológica de outras variáveis intervenientes que causam confusão na interpretação dos dados, como a idade cronológica, a estatura e a composição corporal.

Nedeljkovic et al. ${ }^{6}$ verificaram o efeito da maturação biológica sobre o tamanho corporal e sua relação com o desempenho em vários testes de FM. Os resultados revelaram que existe um incremento no desempenho da FM concomitante com o aumento do tamanho corporal durante a puberdade. Esse incremento é superior aos encontrados durante a pré-puberdade e pós-puberdade. Neu et al. ${ }^{37}$ analisaram o efeito da força de preensão manual em 185 mulheres e 181 homens com idades entre 6 e 23 anos. Os resultados demonstraram um aumento da AST e da FM com a idade. Como esperado, incrementos no tamanho muscular e na FM eram acelerados durante a puberdade nos homens, mas não nas mulheres, o que indica uma influência da maturação biológica e do gênero. Contudo, quando os dados de FM específica (FM/AST) foram ajustados pelo comprimento do antebraço, a FM específica não apresentou diferenças significativas entre os gêneros e demonstrou ser independente dos efeitos dos hormônios sexuais.

Da mesma forma, De Ste Croix et al. ${ }^{3}$ determinaram que a maturação biológica não influencia independentemente o desenvolvimento da FM isocinética de extensores e flexores do joelho quando a estatura e a MC eram devidamente controladas. Os autores ressaltam a necessidade de cautela na interpretação dos dados, porque não existia uma grande variação dos estágios maturacionais. Adicionalmente, Tonson et al. ${ }^{13}$ verificaram o efeito do crescimento e da maturação biológica sobre a FM isométrica em 14 pré-púberes, 16 adolescentes e 16 homens. Os dados foram normalizados por AST, VM e VM estimado por antropometria. Os resultados apresentaram diferenças entre os grupos quando a FM era normalizada por AST e VM estimado; no entanto, não apresentavam diferenças quando normalizados por VM. Os autores concluíram que o VM é a melhor variável de normalização dos dados de FM durante o crescimento e maturação biológica.

O VM tem demonstrado ser essencial para analisarmos o desempenho muscular e suas adaptações em resposta ao treinamento, desuso ou envelhecimento ${ }^{38}$. A máxima capacidade de geração de força do músculo é proporcional a ASTF, podendo ser estimada a partir do VM e do comprimento do fascículo (FL). Assim, o VM é um determinante de massa muscular, tornando-se um parâmetro importante para avaliar a FM do músculo $^{39}$. Atualmente, tem sido utilizado como uma variável de normalização da $\mathrm{FM}^{13}$ e do $\mathrm{TM}^{13,14,40}$. Adicionalmente, essa normalização (FM/VM e TM/VM) tem sido considerada um índice de tensão específica do músculo esquelético ${ }^{39}$.

\section{RECOMENDAÇÕES PARA NORMALIZAÇÃO DA FM E TM}

Existem três questões básicas que devem ser primordialmente consideradas quando comparamos os valores de FM e TM entre crianças, adolescentes e adultos.

$1^{a}$ Identificar a relação dos valores absolutos de FM (geralmente $\mathrm{kg}$ ) e TM ( $\mathrm{Nm})$ com a MC $(\mathrm{kg})$. Para realizarmos essas análises, podemos utilizar a correlação de Pearson (figura 1). Para utilizarmos a MC como variável de normalização, é necessário que exista uma correlação significativa com a FM e TM $(\mathrm{p}<0,05)$. Caso contrário, a FM e TM serão consideradas independentes da MC. Geralmente esse pressuposto 
é atendido na maioria dos estudos.

$2^{a} \mathrm{O}$ próximo passo é verificar se a normalização padrão da FM (FM/MC) e do TM (TM/MC) é independente da $\mathrm{MC}(\mathrm{kg})$. Para isso, devemos novamente realizar uma correlação de Pearson, mas agora com os dados normalizados de FM $\left(\mathrm{kg}^{\mathrm{kg}}{ }^{-1}\right)$ e TM $\left(\mathrm{Nm} \cdot \mathrm{kg}^{-1}\right)$ com a MC (kg). Não havendo correlação significativa, a FM e o TM foram devidamente normalizados. Entretanto, essa expressão raramente tem demonstrado ser independente da MC, geralmente apresentando uma correlação significativa com a MC (figura 2).

$3^{\text {a }}$ Para solucionar essa questão, podemos utilizar a equação alométrica $Y=a X^{b}$. Essa equação pode ser expressa como Log (FM ou TM) = Log a + b Log MC, para dados que apresentarem grande variabilidade. Ela nos permite identificar o expoente alométrico b, que quantifica a relação entre FM ou TM e MC. Resolvemos essa equação utilizando uma regressão linear com a FM absoluta $(\mathrm{kg})$ ou TM absoluto $(\mathrm{Nm})$, representando a variável dependente, e a $\mathrm{MC}$ $(\mathrm{kg})$, a variável independente. Conhecido o valor do expoente $b$, utilizaremos esse valor para expressar a $\mathrm{MC}(\mathrm{kg})$ em uma função potência (ex.: $\mathrm{MC}^{\mathrm{b}}$ ). Suponhamos que o valor do expoente para a FM seja $b=0,67$ e para o TM seja $b=0,75$. O próximo passo é dividir o valor da FM absoluta pela $\mathrm{MC}^{0,67}$; e, da mesma forma, dividir o valor TM absoluto pela $\mathrm{MC}^{0,75}$, criando as expressões alométricas FM (kg.kg${ }^{0,67}$ ) e TM (Nm.kg-0,75). Teoricamente, agora os dados foram devidamente normalizados e identificamos um índice de normalização estatisticamente independente da MC $(p>0,05)$. A figura 3 ilustra esse procedimento.

Nesse sentido, o efeito do tamanho corporal foi devidamente ajustado e a comparação entre indivíduos heterogêneos em sexo, idade, maturação biológica, nível de condicionamento físico e composição corporal (\% gordura, \% massa muscular e $\%$ ósseo) podem ser comparados adequadamente.

\section{CONSIDERAÇÕES FINAIS}

A alometria tem demonstrado ser a metodologia mais adequada para normalizar os dados de FM e TM. Em crianças e adolescentes, essa metodologia
A

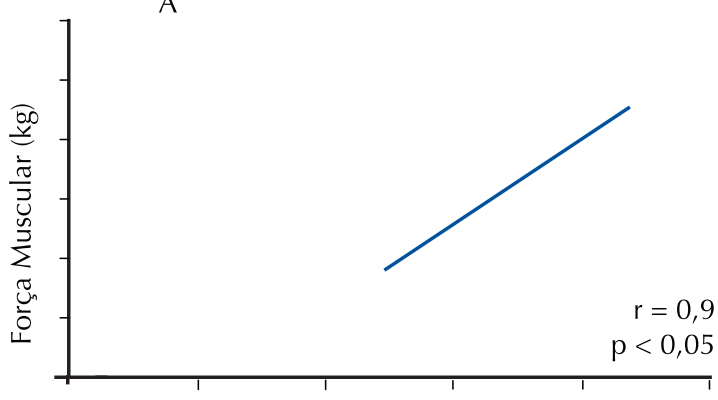

Massa corporal $(\mathrm{kg})$
B

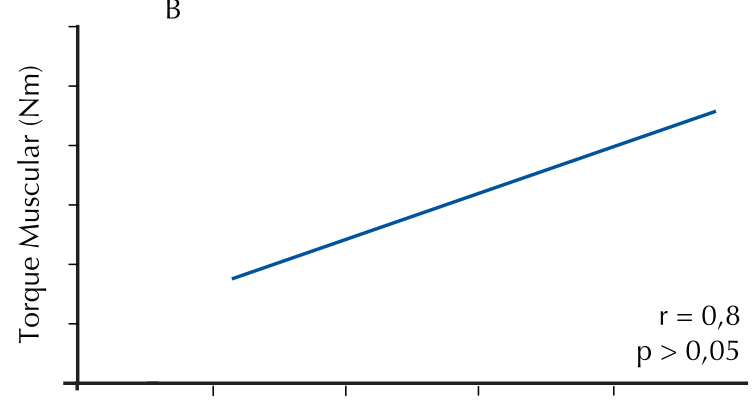

Massa corporal $(\mathrm{kg})$

Figura 1. Regressão linear entre força muscular absoluta e massa corporal (A) e regressão linear entre torque muscular absoluto e massa corporal (B).

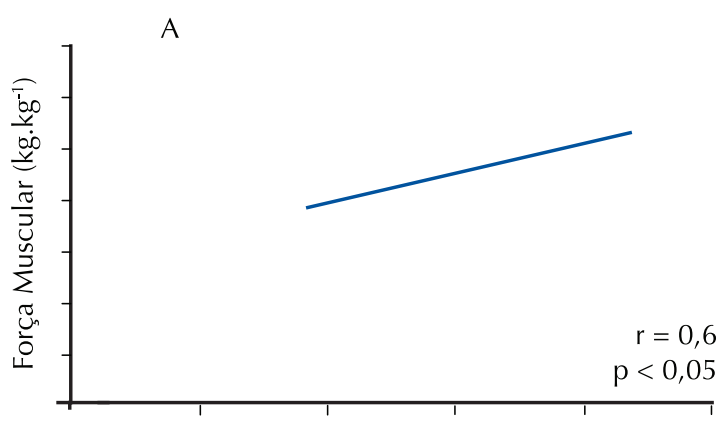

Massa corporal $(\mathrm{kg})$

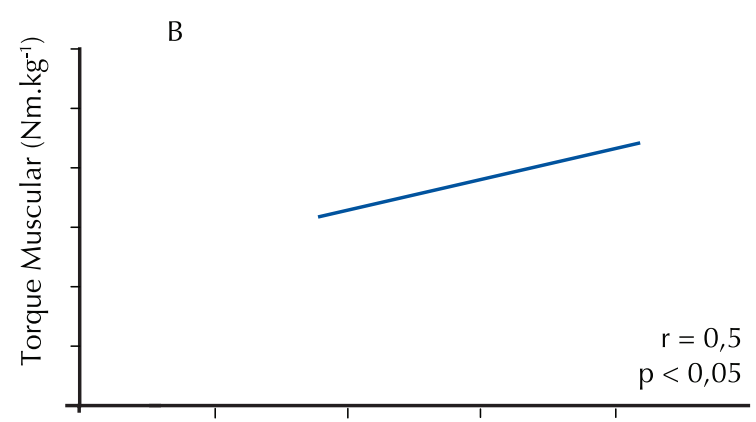

Massa corporal $(\mathrm{kg})$

Figura 2. Regressão linear entre força muscular relativa e massa corporal (A) e regressão linear entre torque muscular relativo e massa corporal (B). 


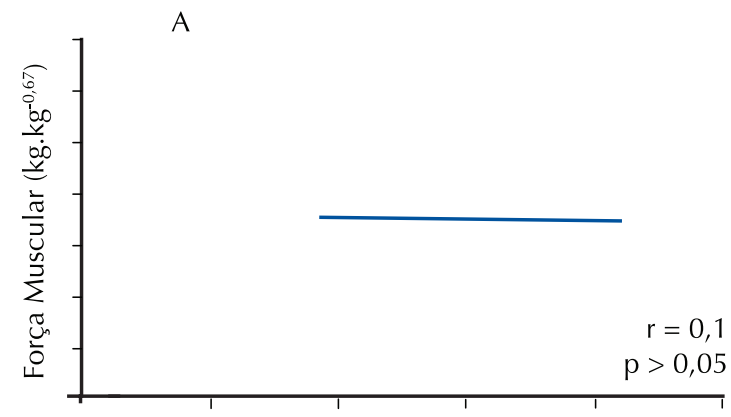

Massa corporal $(\mathrm{kg})$

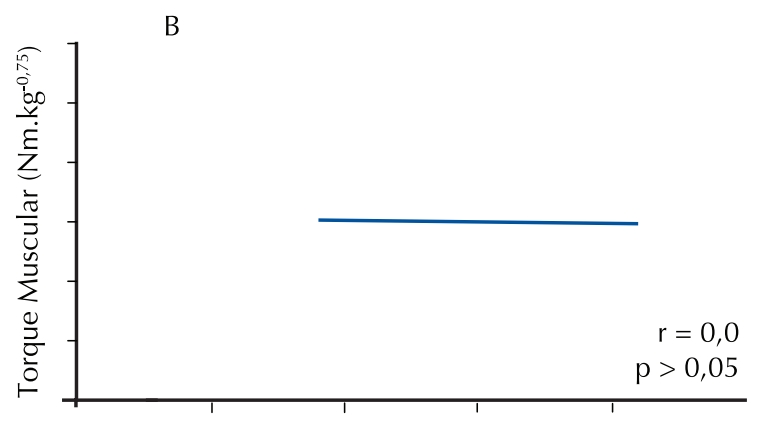

Massa corporal $(\mathrm{kg})$

Figura 3. Regressão linear entre força muscular alométrica e massa corporal (A) e regressão linear entre torque muscular alométrico e massa corporal (B).

também tem demonstrado ser a mais eficiente para a normalização dos dados, além de ser sensível para verificar os efeitos do crescimento e da maturação biológica.

Recomendamos o cálculo dos expoentes alométricos específicos para a FM e TM, verificando se esses estabelecem um índice independente do tamanho corporal.

O VM poderá assumir um papel importante na normalização da FM e TM, pois tem sido considerado superior à MC, MLG e AST como variável de normalização.

\section{Agradecimentos}

Ao CNPq, FINEP e CAPES pelo apoio financeiro a este trabalho.

\section{REFERÊNCIAS BIBLOGRÁFICAS}

1. Malina RM. Weight training in youth-growth, maturation, and safety: an evidence-based review. Clin J Sport Med 2006;16(6):478-87.

2. Hoff J, Helgerud J. Endurance and strength training for soccer players: physiological considerations. Sports Med 2004;34(3):165-80.

3. De Ste Croix MB, Armstrong N, Welsman JR, Sharpe P. Longitudinal changes in isokinetic leg strength in 10-14-year-olds. Ann Hum Biol 2002;29(1):50-62.

4. Rowland TW. Children's Exercise Physiology. $2^{a}$ edition ed: Human Kinetics; 2005.

5. De Ste Croix M, Deighan M, Armstrong N. Assessment and interpretation of isokinetic muscle strength during growth and maturation. Sports Med 2003;33(10):727-43.

6. Nedeljkovic A, Mirkov DM, Kukolj M, Ugarkovic D, Jaric S. Effect of maturation on the relationship between physical performance and body size. J Strength Cond Res 2007;21(1):245-50.

7. Ford P, De Ste Croix M, Lloyd R, Meyers R, Moosavi $\mathrm{M}$, Oliver J, et al. The long-term athlete development model: physiological evidence and application. J Sports Sci 2011;29(4):389-402.

8. Wisloff U, Helgerud J, Hoff J. Strength and endurance of elite soccer players. Med Sci Sports Exerc 1998;30(3):462-7.

9. Jaric S, Radosavljevic-Jaric S, Johansson H. Muscle force and muscle torque in humans require different methods when adjusting for differences in body size. Eur J Appl Physiol 2002;87(3):304-7.

10. Nevill AM, Holder RL, Baxter-Jones A, Round JM, Jones DA. Modeling developmental changes in strength and aerobic power in children. J Appl Physiol 1998;84(3):963-70.

11. Weir JP, Housh TJ, Johnson GO, Housh DJ, Ebersole KT. Allometric scaling of isokinetic peak torque: the Nebraska Wrestling Study. Eur J Appl Physiol Occup Physiol 1999;80(3):240-8.

12. Klein CS, Rice CL, Marsh GD. Normalized force, activation, and coactivation in the arm muscles of young and old men. J Appl Physiol 2001;91(3):1341-9.

13. Tonson A, Ratel S, Le Fur Y, Cozzone P, Bendahan D. Effect of maturation on the relationship between muscle size and force production. Med Sci Sports Exerc 2008;40(5):918-25.

14. Barrett U, Harrison D. Comparing muscle function of children and adults: Effects of scaling for muscle size. Pediatr Exerc Sci 2002;14(4):369-76.

15. Huxley JS. On the relation between egg weight and body weight in birds. JLinn Soc Zool. 1927;36:457-66.

16. Zoeller RF, Ryan ED, Gordish-Dressman H, Price TB, Seip RL, Angelopoulos TJ, et al. Allometric scaling of biceps strength before and after resistance training in men. Med Sci Sports Exerc 2007;39(6):1013-9.

17. Wren TA, Engsberg JR. Normalizing lower-extremity strength data for children without disability using allometric scaling. Arch Phys Med Rehabil 2007;88(11):1446-51.

18. Jaric S. Muscle strength testing: use of normalisation for body size. Sports Med 2002;32(10):615-31.

19. Jaric S. Role of body size in the relation between muscle strength and movement performance. Exerc Sport Sci Rev 2003;31(1):8-12.

20. Jaric S, Mirkov D, Markovic G. Normalizing physical 
performance tests for body size: a proposal for standardization. J Strength Cond Res 2005;19(2):467-74.

21. Markovic G, Jaric S. Movement performance and body size: the relationship for different groups of tests. Eur J Appl Physiol 2004;92(1-2):139-49.

22. Markovic G, Jaric S. Scaling of muscle power to body size: the effect of stretch-shortening cycle. Eur J Appl Physiol 2005;95(1):11-9.

23. Folland JP, Mc Cauley TM, Williams AG. Allometric scaling of strength measurements to body size. Eur J Appl Physiol 2008;102(6):739-45.

24. Vanderburgh PM, Mahar MT, Chou CH. Allometric scaling of grip strength by body mass in college-age men and women. Res Q Exerc Sport 1995;66(1):80-4.

25. Baroni BM, Leal Junior EC, De Marchi T, Lopes AL, Salvador M, Vaz MA. Low level laser therapy before eccentric exercise reduces muscle damage markers in humans. Eur J Appl Physiol 2010;110(4):789-96.

26. Cometti G, Maffiuletti NA, Pousson M, Chatard JC, Maffulli N. Isokinetic strength and anaerobic power of elite, subelite and amateur French soccer players. Int J Sports Med 2001;22(1):45-51.

27. Hulens M, Vansant G, Lysens R, Claessens AL, Muls E, Brumagne S. Study of differences in peripheral muscle strength of lean versus obese women: an allometric approach. Int J Obes Relat Metab Disord 2001;25(5):676-81.

28. Fukunaga T, Miyatani M, Tachi M, Kouzaki M, Kawakami Y, Kanehisa H. Muscle volume is a major determinant of joint torque in humans. Acta Physiol Scand 2001;172(4):249-55.

29. Jones EJ, Bishop PA, Woods AK, Green JM. Cross-sectional area and muscular strength: a brief review. Sports Med 2008;38(12):987-94.

30. Maughan RJ, Watson JS, Weir J. Relationships between muscle strength and muscle cross-sectional area in male sprinters and endurance runners. Eur J Appl Physiol Occup Physiol 1983;50(3):309-18.

31. Schmidt-Nielsen k. Scaling: Why is animal size so important. Cambrige :Cambrige Press; 1984.

32. Cunha GS, Célia FG, Ribeiro JL, Oliveira AR. Effects of the biological maturation on maximal oxygen uptake and ventilatory breakpoint of Brazilian soccer players. Gazz Med Ital - Arch Sci Med 2008;167(2):43-9.

33. Cunha GD, Lorenzi TD, Sapata KB, Lopes AL, Gaya ACA, Oliveira AR. Effect of biological maturation on maximal oxygen uptake and ventilatory thresholds in soccer players: An allometric approach. J Sports Sci 2011;29(10):1129-39.

34. Davies MJ, Dalsky GP. Normalizing strength for body size differences in older adults. Med Sci Sports Exerc 1997;29(5):713-7.

35. De Ste Croix MBA, Armstrong N, Welsman JR. Concentric isokinetic leg strength in pre-teen, teenage and adult males and females. Biol Sport 1999;16:75-86.

36. Aasa U, Jaric S, Barnekow-Bergkvist M, Johansson $\mathrm{H}$. Muscle strength assessment from functional performance tests: role of body size. J Strength Cond Res 2003;17(4):664-70.

37. Neu CM, Rauch F, Rittweger J, Manz F, Schoenau E. Influence of puberty on muscle development at the forearm. Am J Physiol Endocrinol Metab 2002;283(1):E103-7.

38. Reeves ND, Maganaris CN, Narici MV. Ultrasonographic assessment of human skeletal muscle size. Eur J Appl Physiol 2004;91(1):116-8.

39. Lynch NA, Metter EJ, Lindle RS, Fozard JL, Tobin JD, Roy TA, et al. Muscle quality. I. Age-associated differences between arm and leg muscle groups. J Appl Physiol 1999;86(1):188-94.

40. Thom JM, Morse CI, Birch KM, Narici MV. Influence of muscle architecture on the torque and power-velocity characteristics of young and elderly men. Eur J Appl Physiol 2007;100(5):613-9.

\section{Endereço para correspondência}

Giovani dos Santos Cunha

Endereço: Rua Felizardo, n 750 ,

ESEF/UFRGS-LAPEX

Porto Alegre - RS - Brasil

Cep: $90690-200$

Email: giovanicunha@yahoo.com.br 